\begin{tabular}{|c|l|}
\hline Title & Effects of eye position on estimates of eye displacement for spatial updating. \\
\hline Author(s) & Tanaka, Masaki \\
\hline Citation & NeuroReport, 16(12), 1261-1265 \\
\hline Issue Date & 2005-08_22 \\
\hline Doc URL & http://hdl.handle.net/2115/43047 \\
\hline Rights & $\begin{array}{l}\text { Thisisa non-final version of an article published in final form in } \\
\text { (http://journals.Iww.com/heuroreport/Abstract/2005/08220/Effects_of_eye_position_on_estimates_of_eye.2.aspx) }\end{array}$ \\
\hline Type & article (author version) \\
\hline File Information & NR16_1.pdf \\
\hline
\end{tabular}

Instructions for use 


\title{
Effects of eye position on estimates of eye dis placement for spatial updating
}

\author{
Masaki Tan aka
}

Department of Physiology, Hokkaido University School of Medicine, Sapporo 060-8638, Japan

Running head: Effects of eye position on spatial updating

Number of figures: 4

Abstract: 120 words

Number of References: 25

Text (excluding References and Captions): 13,227 characters with space

Estimate of actual figure size: 4,750 characters (35 cm in single column)

Subtotal: 17,977 characters

Captions: 2,124 characters with space

Correspondence to:

Masaki Tanaka

Department of Physiology

Hokkaido University School of Medicine

North 15, West 7, Sapporo 060-8638, JAPAN

Tel: $\quad+81117065040$

FAX: +81117065041

E-mail:masaki@med.hokudai.ac.jp.

Supported by grants from the MEXT of Japan. 


\section{ABST RACT}

Neurons in the parietal cortex represent spatial memory of visual stimuli in an eye-centered coordinate frame. To preserve spatial stability across eye movements, spatial memory must be updated during each eye movement. Since eye movement signals in the parietal cortex are known to be modulated by eye position in the orbit, estimates of eye displacement based on these signals could also be influenced by eye position. The present study examined the possible effect of eye position on the accuracy of memory-guided saccades in monkeys following saccades or smooth pursuit during the memory period. The results showed that eye position has a modest effect on saccade localization, suggesting that eye position signal plays a modulatory role in updating visuospatial working memory.

Key words: Saccade; Smooth pursuit; Spatial memory; Mislocalization; Eye position; Eye movements; Monkey 


\section{INT RODUCTION}

The ability to preserve spatial stability across self-motions is essential for goal-directed behavior. Previous studies have shown that both humans and monkeys are able to maintain spatial stability during saccadic [1-5] as well as smooth pursuit eye movements [5-8]. Most of these studies showed that, unless visual stimuli were presented during or around the time of eye movements, saccades to the remembered location of a previously presented visual stimulus were quite accurate. Although the parietal cortex has been shown to play a central role $[9,10]$, how the brain maintains spatial stability remains unclear. Since many neurons in the parietal cortex represent visual space in eye-centered $[11,12]$ rather than head-centered coordinate frames [13], the brain must update spatial memory by monitoring each eye movement during the memory period. Recent studies have proposed two different models that used different signals for this purpose. In one model, intervening eye movements are computed based on changes in eye position signals [14]. In the other, both eye velocity and eye position signals are used, while velocity signals play a more important role than position signals [15]. The purpose of the present study was to examine the possible effects of orbital eye positions on the estimates of eye displacement expected with both models.

Assuming that the brain compares eye position signals before and after eye movements, the accuracy of estimates of eye displacement would rely on the linearity of eye position signals. It remains uncertain, however, whether eye positions are linearly represented in the cerebral cortex. Previous studies showed that individual neurons in parietal area V6 discharged during fixation in a restricted eye-position field, without showing planar representation of eye position $[16,17]$. In addition, all visual, saccade, memory, and background activity of parietal neurons is known to be strongly affected by eye position, and the relationship between eye position and the gain modulation of these properties includes non-linear elements for many neurons [11]. On the other hand, if the brain monitors eye velocity signals, the gain modulation of saccade signals in the parietal cortex would also predict the effects of eye position on estimates of eye displacement. It 
has also been reported that neurons in the parietal $[18,19]$ and frontal $[20]$ cortices modulate their pursuit-related activity depending on eye position. Thus, available data suggest that estimates of eye displacement could be affected by eye position, regardless of the signals used.

The present study examines the possible effects of orbital eye position on the accuracy of memory-guided saccades in monkeys following saccades or smooth pursuit during the delay period. Care was taken to match the amplitude of saccades, duration of the delay period, and retinal eccentricity of visual stimuli between trials, while the direction of intervening eye movements and initial fixation location were varied from trial to trial. The results show that eye position has a modest effect on saccade localization, suggesting that eye position signals play a role in updating visuospatial memory.

\section{MATERIALS AND MET HODS}

Animal preparation: Three adult Japanese monkeys (Macaca fuscata, 6-12 kg) were used. Two participated in previous behavioral experiments [21]. All experimental protocols were approved in advance by the Animal Care and Use Committee of the Hokkaido University School of Medicine and were in accordance with the NIH Guide for the Care and Use of Laboratory Animals. Animals were prepared for chronic experiments by implanting a head-holding device and eye coil under general anesthesia and using sterile procedures. Animals received analgesia after each surgical procedure. Water intake was controlled daily so that monkeys were motivated to perform oculomot or tasks.

Visual stimuli and behavioral tasks: Experiments were controlled by a Windows-based real-time data acquisition system (TEMPO, Reflective Computing). Visual stimuli were presented on a 24-inch cathode-ray tube monit or (SONY GDM-FW900, refresh rate: $60 \mathrm{~Hz}$ ) that was located $38 \mathrm{~cm}$ away from the eyes and subtended $64 \times 44^{\circ}$ of visual angle. A $0.5^{\circ}$ square spot served as the visual stimulus. Experiments were carried out in a darkened booth. Eye position signals were calibrated before each experiment by having monkeys fixate a 
stationary target spot at known visual angles. Thereafter, visual stimuli were presented in individual trials, and monkeys were reinforced with drops of apple juice for each correct trial.

Each trial began with the onset of a fixation target placed along the horizontal meridian. The horizontal location of the initial fixation target varied from trial to trial, and was chosen randomly at intervals of $2^{\circ}$ within a range of $\pm 32^{\circ}$ (Experiment $\# 1$ ) or $\pm 18-20^{\circ}$ (Experiment \#2). In Experiment \#1 (Figs.1a and b), a white target spot ('cue') appeared briefly (200 ms) during fixation of $1,100-1,400 \mathrm{~ms}$. The location of the cue was within $\pm 23^{\circ}$ of the screen center, and 9,12 , or $15^{\circ}$ horizontal to and $10^{\circ}$ above the red fixation target. After an additional 650 (saccade paradigm) or $300 \mathrm{~ms}$ (pursuit paradigm) fixation interval, the fixation target was moved toward the cue location along the horizontal meridian. In the saccade paradigm, the target was displaced by $12^{\circ}$ and remained visible for $750 \mathrm{~ms}$. In the pursuit paradigm, the target jumped $3^{\circ}$ then moved toward the fixation location at $20 \%$ s (step-ramp target motion). After a motion period of $750 \mathrm{~ms}$, the target became stationary and remained visible for $350 \mathrm{~ms}$. In both paradigms, animals were required to maintain their eye position within $3^{\circ}$ of the target (except during a 400-ms period at the time of target step) until it disappeared $1400 \mathrm{~ms}$ after offset of the cue, then to make a memory-guided saccade toward the previously flashed cue location within $400 \mathrm{~ms}$. To motivate monkeys to make accurate saccades, the cue reappeared at the end of each trial. Animals obtained a reward when their eye position was within $8^{\circ}$ horizontal and $6^{\circ}$ vertical of the cue location at the time of cue reappearance. In Experiment \#2 (Figs.1a and c), a green fixation target jumped horizontally (saccade paradigm, $12^{\circ}$ ) or moved in a step-ramp fashion (pursuit paradigm, $-3^{\circ}$ step and $20 \% \mathrm{~s} \mathrm{ramp}$ ) for $750 \mathrm{~ms}$. In the saccade paradigm, the target disappeared either immediately (no delay) or 1,000 ms (with delay) after the eye position entered within $4^{\circ}$ of the target location. In the pursuit paradigm, the target disappeared $250 \mathrm{~ms}$ after the offset of target motion. Animals were required to make a memory-guided saccade toward the location of the initial fixation before the target reappeared 600 (saccade paradigm without delay) or $400 \mathrm{~ms}$ (other tasks) later. Again, monkeys received a reward when their eye position was within $8^{\circ}$ horizontal and $3^{\circ}$ vertical of the initial fixation location at the time of 
target reappearance. Experiments \#1 and\#2 were carried out in separate blocks.

Data acquisition and analysis: Horizontal and vertical eye position signals were digitized at $1 \mathrm{kHz}$ and stored in files during experiments with other codes of task events. Data were analyzed off-line using Matlab (Mathworks). The accuracy of memory-guided saccades was assessed by measuring the difference in the horizontal target location and eye position at the time of target reappearance (closed triangles in Figs. $1 \mathrm{~b}$ and c). Values were compared with the horizontal eye position at the time of target offset (Experiment \#1, open triangles in Fig. 1B) or time of target motion onset (Experiment $\# 2$, open triangles in Fig. 1c). Linear regression analyses were performed for each set of data.

\section{RESULTS}

In Experiment \#1, the accuracy of memory-guided saccades was affected by both eye position and the direction of eye movements during the delay period. Fig. 2 shows the amount of mislocalization of individual saccades as a function of eye position at the time of fixation target offset. In both the saccade and pursuit paradigm (Figs. 2a and b, respectively), the direction and magnitude of mislocalization depended on eye position. When the fixation target disappeared at eccentric locations, vertical memory-guided saccades tended to be directed to a more central location than the actual location of the cue, irrespective of the direction and kind of intervening eye movements. Type II regression lines fitted to the data had slopes ranging from -0.12 to -0.07 (mean, $-0.10 \pm 0.02)$ that were statistically different from zero $(p \varangle 0.0001)$ in all four cases. Fig. $4 \mathrm{a}$ summarizes the regression slopes for three animals. Average values were $-0.07 \pm$ 0.04 and $-0.06 \pm 0.04$ for the saccade and pursuit paradigms, respectively. In addition to the effects of eye position, the accuracy of saccades was also affected by the direction of intervening eye movements. The endpoints of saccades were shifted in the direction of prior eye movements as shown by different colors in Fig. 2. Differences in the y-intercept of the regression lines for trials in different directions (leftward minus rightward) were -2.08 and 
$-2.19^{\circ}$ for the saccade and pursuit paradigms, respectively. Fig. $4 \mathrm{~b}$ shows the values for the three monkeys that averaged $-0.99 \pm 1.01^{\circ}$ for saccade and $-2.60 \pm 0.77^{\circ}$ for pursuit.

The effects of eye position were also evident when animals made a saccade toward the location of the initial fixation in Experiment \#2. Fig. 3 shows the errors of individual memory-guided saccades as a function of eye position during the initial fixation for the three different paradigms. As above, memory-guided saccades tended to be directed to a more central location when the initial fixation target appeared peripherally. In the saccade paradigms (Figs. $3 a$ and $b$ ), the duration of the delay period had virtually no effect, and the type II regression slopes for each saccade direction ranged from -0.05 to -0.07 . In the pursuit paradigm (Fig. $3 \mathrm{c}$ ), the effects of eye position were much greater, and the regression slopes were -0.26 and -0.24 for right ward and leftward pursuit, respectively. The values of regression slopes are summarized in Fig. $4 \mathrm{a}$ for all three animals that averaged $-0.11 \pm 0.04$ (saccade without delay), $-0.09 \pm 0.07$ (saccade with delay) and $-0.16 \pm 0.08$ (pursuit). On the other hand, the effects of prior eye movement direction were absent or even in the opposite direction in Experiment \#2. For example, shifts in the regression line y-intercept (leftward minus rightward) were $-0.26^{\circ}$ (saccades without delay), $0.57^{\circ}$ (saccades with a delay) and $-0.5^{\circ}$ (pursuit) for the data shown in Fig.3. Average values for the three monkeys were $-0.22 \pm 0.04^{\circ}$ (saccade without delay), $1.02 \pm$ $0.63^{\circ}$ (saccade with delay) and $-0.23 \pm 0.25^{\circ}$ (pursuit).

\section{DISCUSSION}

The present study examined the ability of the visual system to update spatial memory during eye movements. To avoid perisaccadic mislocalization of visual stimuli, which has been analyzed in many previous studies [3,22-24], this study introduced a delay period between stimulus presentation and eye movements (Experiment \#1) or required monkeys to remember the initial fixation location without presenting any explicit visual cue (Experiment \#2). The results showed that the accuracy of spatial updating during eye movements relies on at least two factors. 
Firstly, the accuracy of saccades depended on the direction of intervening eye movements. In Experiment \#1, the endpoint of saccades was systematically shifted in the direction of prior eye movements. This effect, however, was greatly reduced or diminished when monkeys were required to make a saccade to the location of the initial fixation (Experiment \#2). This could be due to the fact that both visual and eye movement signals are necessary for computing the goals of saccades in Experiment \#1, while eye movement signals only are sufficient in Experiment \#2. The interaction between logarithmic (visual) and almost linear (eye position) coordinate frames might result in this directional effect, as suggested by a recent study that simulated perisaccadic mislocalization [25].

The second and more consistent factor affecting saccade accuracy was eye position in the orbit. Regardless of the direction of intervening eye movements, the endpoint of saccades was shifted toward a more central location within the oculomotor range. As a result, the direction of mislocalization was reversed with eccentric eye positions. The origin of this eye position effect is unknown. It might have been inherited from the non-linearity of eye position signals in the cerebral cortex, as pointed out above, or alternatively, if eye displacement is computed based on eye velocity rather than eye position signals [15], the gain modulation of eye movement signals in the parietal cortex $[11,18,19]$ might result in the eye position effect.

In summary, the present study showed that the accuracy of memory-guided saccades is affected modestly by both the direction of intervening eye movements and position of the eyes in the orbit. The latter effect suggests that eye position signals play a modulatory role in monitoring eye displacement for update of the visuospatial working memory. 


\section{Acknowledgements}

I thank S. Hirano for technical assistance. One monkey was provided by the Primate Research Institute of the Kyoto University. This work was supported by a grant-in-aid for scientific research on priority areas ("Advanced Brain Science Project”) and a grant for young scientists from the MEXT of Japan. 


\section{REFERENCES}

1. Hallett PE and Lightstone AD. Saccadic eye movements to flashed targets. Vision Res 1976; 16:107-114.

2. Mays LE and Sparks DL. Saccades are spatially, not retinocentrically, coded. Science 1980; 208:1163-1165.

3. Dassonville P, Schlag J, Schlag-Rey M. Oculomotor localization relies on a damped representation of saccadic eye displacement in human and nonhuman primates. Vis Neurosci 1992; 9:261-269.

4. Karn KS, Moller P and Hayhoe MM. Reference frames in saccadic targeting. Exp Brain Res 1997; 115:267-282.

5. Baker JT, HarperTM and Snyder LH. Spatial memory following shifts of gaze. I. Saccades to memorized world-fixed and gaze-fixed targets. J Neurophysiol 2003; 89:2564-2576.

6. Schlag J, Schlag-Rey M and Dassonville P. Saccades can be aimed at the spatial location of targets flashed during pursuit. J Neurophysiol 1990; 64:575-581.

7. Honda H. The extraretinal signal from the pursuit-eye-movement system: its role in the perceptual and the egocentric localization systems. Percept Psychophys 1990; 48:509-515.

8. Zivotofsky AZ, Rottach KG, Averbuch-Heller L, Kori AA, Thomas CW, Dell'Osso LF and Leigh RJ. Saccades to remembered targets: the effects of smooth pursuit and illusory stimulus motion. J Neurophysiol 1996; 76:3617-3632.

9. Duhamel J-R, Goldberg ME, FitzGibbon EJ, Sirigu A and Grafman J. Saccadic dysmetria in a patient with a right frontoparietal lesion: the importance of corollary discharge for accurate spatial behavior. Brain 1992; 115:1387-1402.

10. Li CS and Andersen RA. Inactivation of macaque lateral intraparietal area delays initiation 
of the second saccade predominantly from contralesional eye positions in a double-saccade task. Exp Brain Res 2001; 137:45-57.

11. Andersen RA, Bracewell RM, Barash S, Gnadt JW and Fogassi L. Eye position effects on visual, memory, and saccade-related activity in areas LIP and 7a of macaque. $J$ Neurosci 1990; 10:1176-1 196.

12. Colby CL and Goldberg ME Space and attention in parietal cortex. Annu Rev Neurosci 1999; 22:319-349.

13. Galletti C, Battaglini PP and Fattori P. Parietal neurons encoding spatial locations in craniotopic coordinates. Exp Brain Res 1993;96:221-229.

14. Xing $\mathrm{J}$ and Andersen RA. Memory activity of LIP neurons for sequential eye movements simulated with neural networks. J Neurophysiol 2000;84:651-665.

15. White RL III and Snyder LH. A neural network model of flexible spatial updating. $J$ Neurophysiol 2004;91:1608-1619.

16. Galletti C, Battaglini PP and Fattori P. Eye position influence on the parieto-occipital area PO (V6) of the macaque monkey. Eur J Neurosci 1995; 7:2486-2501.

17. Nakamura K, Chung HH, Graziano MS and Gross CG Dynamic representation of eye position in the parieto-occipital sulcus. $J$ Neurophysiol 1999; 81:2374-2385.

18. Bremmer F, Ilg UJ, Thiele A, Distler C and Hoffmann KP. Eye position effects in monkey cortex. I. Visual and pursuit-related activity in extrastriate areas MT and MST. $J$ Neurophysiol 1997; 77:944-961.

19. Bremmer F, Distler C and Hoffmann KP. Eye position effects in monkey cortex. II. P ursuitand fixation-related activity in posterior parietal areas LIP and 7A. J Neurophysiol 1997; 77:962-977. 
20. Tanaka M and Lisberger SG Role of arcuate frontal cortex of monkeys in smooth pursuit eye movements. I. Basic response properties to retinal image motion and position. $J$ Neurophysiol 2002; 87:2684-2699.

21. Tanaka M. Contribution of signals downstream from adaptation to saccade programming. $J$ Neurophysiol 2003;90:2080-2086.

22. Ross J, Morrone MC and Burr DC. Compression of visual space before saccades. Nature 1997; 386:598-601.

23. Lappe M, Awater H, Krekelberg B. Postsaccadic visual references generate presaccadic compression of space. Nature 2000; 403:892-895.

24. Kaiser M and Lappe M. Perisaccadic mislocalization orthogonal to saccade direction. Neuron 2004; $41: 293-300$.

25. VanRullen R. A simple translation in cortical log-coordinates may account for the pattern of saccadic localization errors. Biol Cybern 2004; 91:131-137. 


\section{CAPTIONS}

Fig. 1. (a) Behavioral paradigms. The location of initial fixation was varied from trial to trial. (b) Representative examples of eye position traces in Experiment \#1. The fixation target was either displaced (saccade trial, upper traces) or moved smoothly (pursuit trial, lower traces) at the time indicated by downward arrowheads. In each trial, horizontal eye positions were measured at the time of fixation target offset (open triangles) and cue reappearance (filled triangles). (c) Examples of eye movement records in Experiment $\# 2$. Saccades were made toward the initial fixation location in response to offset of the fixation target (downward arrowheads). Eye positions were measured at the end of the initial fixation period (open triangles) and time of target reappearance (filled triangles). HE and VE denote horizontal and vertical eye positions, respectively.

Fig. 2. Results of Experiment \#1 for monkey D in the (a) saccade paradigm and (b) pursuit paradigm. Horizontal saccade endpoints are shown relative to the cue location and plotted as a function of eye position at the time of fixation target offset. Red and blue data points indicate data for trials with rightward and leftward eye movements during the delay period, respectively. Continuous lines indicate the type II regression lines for each data set.

Fig. 3. Results of Experiment $\# 2$ for monkey A. Panels show the amounts of mislocalization of return saccades as a function of initial fixation location for (a) the saccade paradigm without a delay, (b) the saccade paradigm with a 1000-ms delay and (c) the pursuit paradigm. Red and blue symbols indicate data for rightward and leftward eye movements during the delay period, respectively.

Fig. 4. Quantitative summary. (a) Effect of eye position in all paradigms. Data points show the slope of type II regression lines fitted to the amount of mislocalization and eye position. Different symbols indicate data from different animals. (b) Effect of eye movement direction in all paradigms. Data points show differences in the y-intercepts of regression 
lines for eye movements in opposite directions. 
(a)

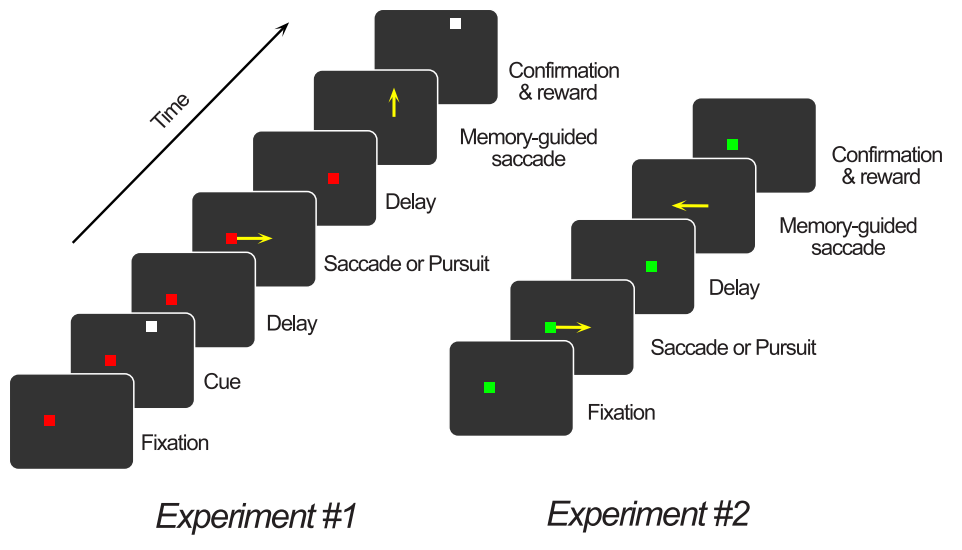

(b) Experiment \#1

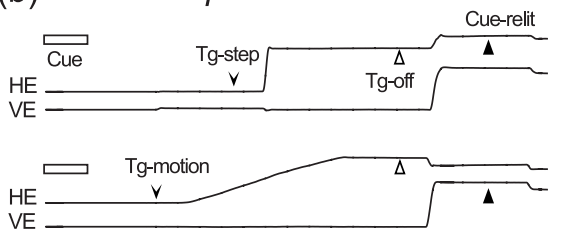

(c) Experiment \#2

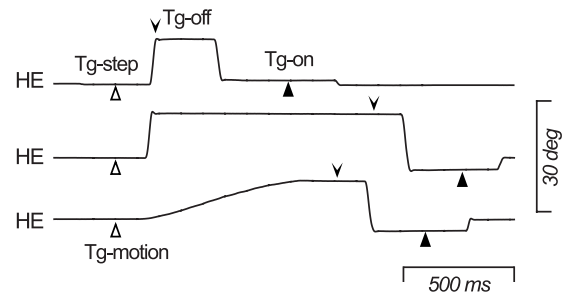

Figure 1, Tanaka 

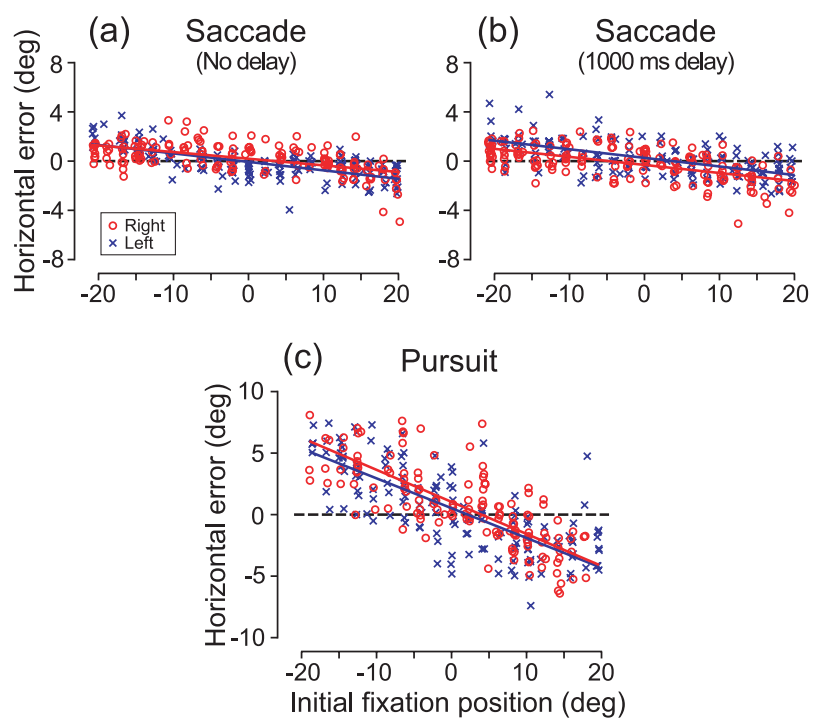

\section{Figure 3, Tanaka}



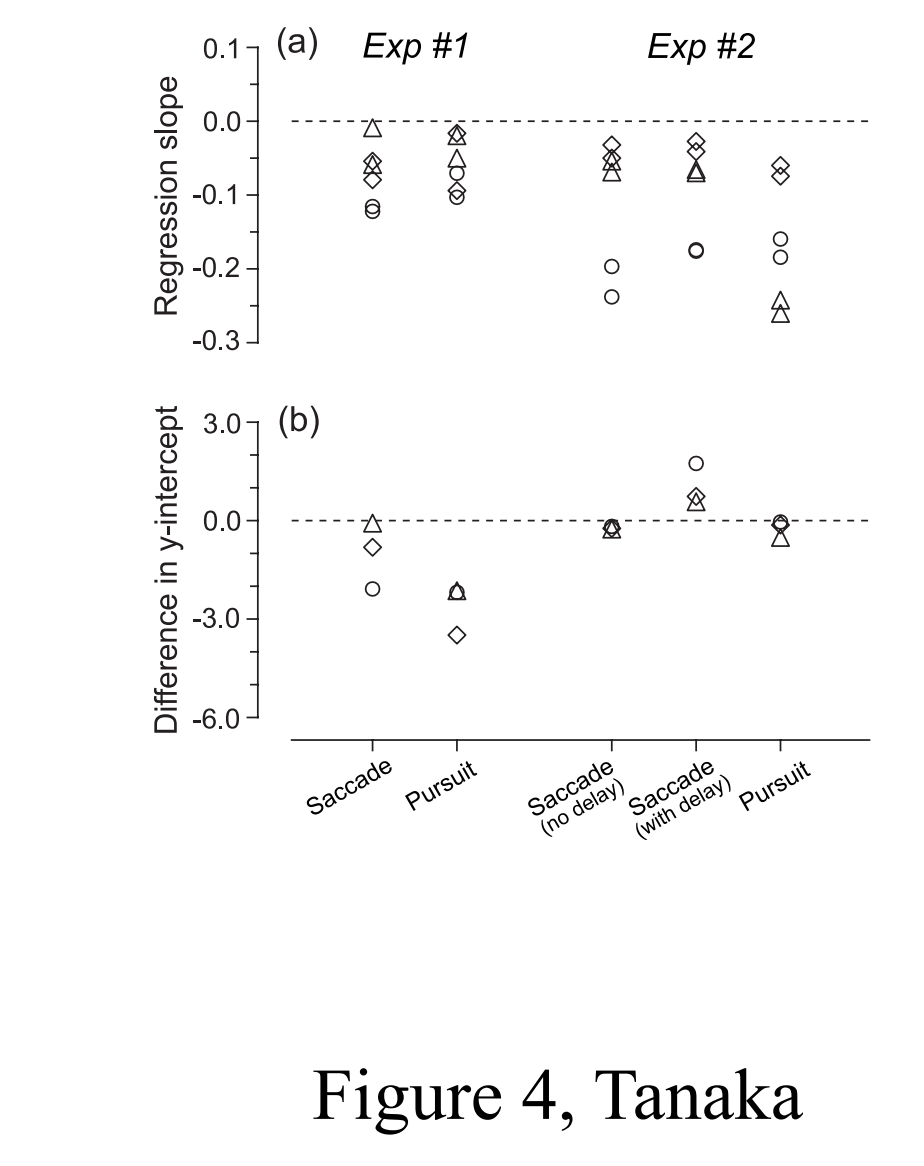

\title{
Figure Four_b/w
}

\author{
Figure 4, Tanaka
}

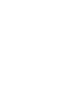
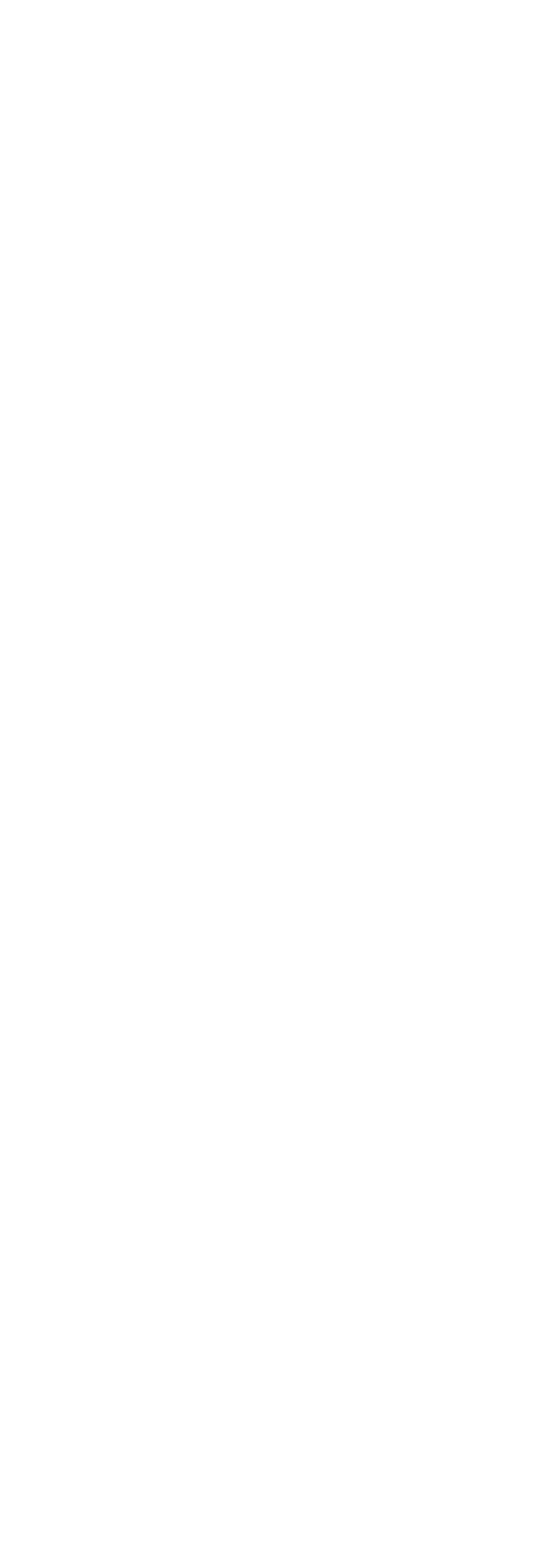

(

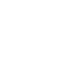
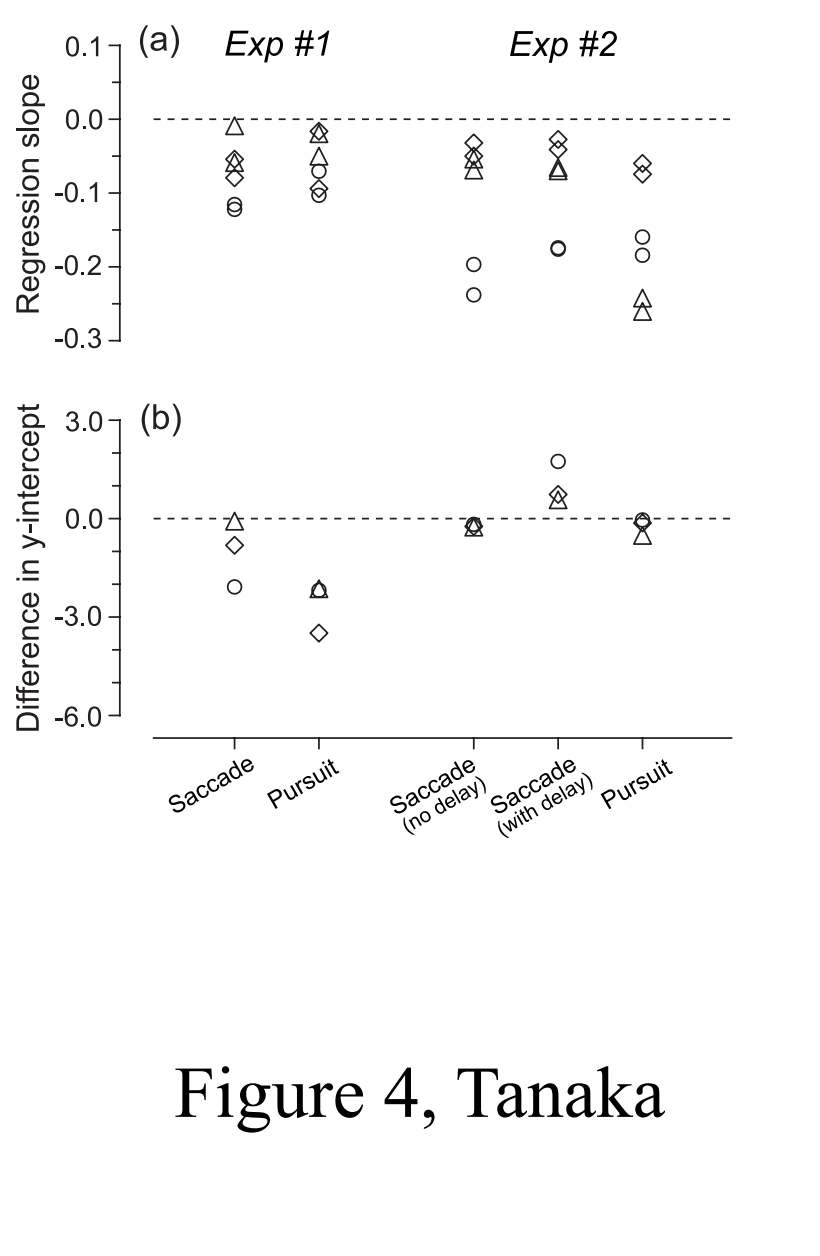